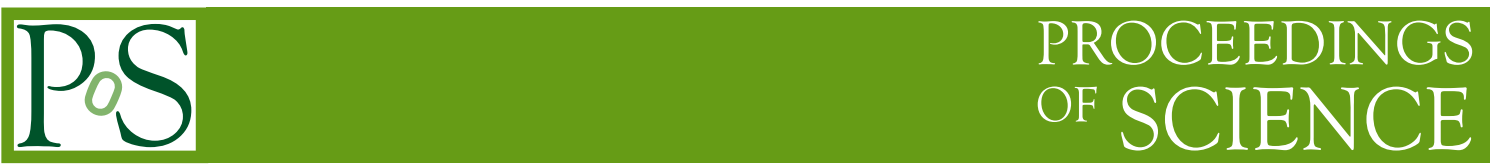

\title{
The Light Dark Matter eXperiment (LDMX)
}

\section{Omar Moreno* for the LDMX Collaboration}

a SLAC National Accelerator Laboratory, Stanford University, Stanford, CA 94309, USA

E-mail: omorenoeslac.stanford.edu

The Light Dark Matter eXperiment (LDMX) proposes a high-statistics search for low-mass dark matter in fixed-target electron-nucleus collisions. Ultimately, LDMX will explore thermal relic dark matter over most of the viable sub-GeV mass range to a decisive level of sensitivity. To achieve this goal, LDMX employs the missing momentum technique, where electrons scattering in a thin target can produce dark matter via "dark bremsstrahlung" giving rise to significant missing momentum and energy in the detector. To identify these rare signal events, LDMX individually tags incoming beam-energy electrons, unambiguously associates them with low energy, moderate transverse-momentum recoils of the incoming electron, and establishes the absence of any additional forward-recoiling charged particles or neutral hadrons. LDMX will employ low mass tracking to tag incoming beam-energy electrons with high purity and cleanly reconstruct recoils. A high-speed, granular calorimeter with MIP sensitivity is used to reject the high rate of bremsstrahlung background at trigger level while working in tandem with a hadronic calorimeter to veto rare photo-nuclear reactions.

XXXIX International Conference on High Energy Physics

4-11 July 2018

Seoul, South Korea

${ }^{*}$ Speaker. 


\section{Introduction}

The existence of dark matter (DM) has been firmly established by the overwhelming evidence from several sources including observations of galactic rotation curves, measurements of the power spectrum of the Cosmic Microwave background, gravitational lensing and galaxy cluster collisions. A comprehensive review can be found in [1]. However, even with all of this evidence, the particle nature of dark matter continues to be one of the most pressing challenges facing particle physics.

One of the simplest possibilities postulates that dark matter arose as a thermal relic from the hot early Universe. Such a scenario only requires a small non-gravitational interaction with the Standard Model (SM) and will lead to the correct thermal relic abundance observed today as long as the DM-SM interaction rate exceeds the Hubble expansion rate at some point in the early Universe. Such a mechanism for generating the thermal DM abundance leads to a minimum annihilation rate $\left(\langle\sigma v\rangle \sim 10^{-26} \mathrm{~cm}^{3} \mathrm{~s}^{-1}\right)$ which is required to avoid production of an overabundance of DM and must be experimentally probed to rule out DM of thermal origin. Furthermore, it also defines a clear mass range $(\sim \mathrm{MeV}$ to $\sim 10 \mathrm{~s} \mathrm{TeV})$ that a thermal DM candidate may have.

Traditionally, searches for thermal DM have focused on Weakly Interacting Massive Particles (WIMPs) with masses between $\sim \mathrm{GeV}-10 \mathrm{TeV}$ that are charged under the electroweak force. Searches for WIMPs by both direct and indirect detection experiments has been motivated by the connection between WIMPs and supersymmetry (SUSY) which also predicts a DM candidate with similar properties. However, decades of searches for WIMPs have overwhelmingly ruled out the simplest WIMP scenarios and next generation experiments (e.g. SuperCDMS, LZ) are either going to rule out or discover WIMP dark matter.

Light (i.e. sub-GeV) dark matter (LDM), in the broad vicinity of the weak scale, is a simple generalization of WIMPs but requires a new force to achieve the correct thermal relic abundance (WIMP's are limited by the Lee-Weinberg Bound [2]). Such a candidate is well-motivated by several "hidden sector" scenarios highlighted in $[3,4]$ and has been the focus of a broad array of searches and experiments for many years now.

As a benchmark scenario, take the simplest hidden sector models where dark matter is charged under a new $U(1)^{\prime}$ gauge field mediated by a $U(1)^{\prime}$ gauge boson $A^{\prime}$ (or "dark photon"). In such models, the dark photon "kinetically mixes" with the $U(1)_{Y}$ (hypercharge) gauge boson with a strength $\varepsilon$, inducing an effective coupling between the dark photon and electric charge. Because of this effective coupling, dark photons can be produced in a process analogous to bremsstrahlung radiation. Assuming that $m_{A^{\prime}}>2 m_{\chi}$, then the dark photon will dominantly decay to DM.

The Light Dark Matter eXperiment (LDMX) will make use of such a production mechanism to conduct a high-statistics search for light dark matter in electron-nucleus collisions using the energy-angle kinematics of the recoiling electron ("missing momentum" technique). It should be noted that while on-shell mediator production often dominates the dark matter signal rate, an on-shell mediator is in no way necessary to produce dark matter in LDMX. In particular, direct production of dark matter through an off-shell mediator is readily visible in LDMX, and is also often important. To identify these rare signal events, LDMX will employ low mass tracking to tag the incoming beam-energy electrons with high purity and cleanly reconstruct recoils. A high-speed, granular calorimeter (ECal) with MIP sensitivity is used to reject the high rate of bremsstrahlung background at trigger level while working in tandem with a hadronic calorimeter ( $\mathrm{HCal}$ ) to veto rare 
photo-nuclear reactions. These proceedings will provide an overview of the LDMX experiment. An initial design study for LDMX can be found in [5].

\section{Dark Matter Signal Production}

As discussed in Section 1, LDMX will employ an electron beam to produce dark photons via the "dark bremsstrahlung" process which subsequently decay to pairs of dark matter particles. The kinematics of the electron in DM production depend on the mass of the $A^{\prime}$. As long as the $A^{\prime}$ or $\chi \bar{\chi}$ pair that is produced is heavy relative to the electron mass, the differential cross-section for DM production is peaked in the phase space where the DM carries away the majority of the beam energy and the electron carries relatively little. This is, of course, the exact opposite of the structure for the kinematics of the vast majority of bremsstrahlung events, and the primary kinematic handle for a missing momentum search. Taking a signal selection of $E_{\text {cut }}=0.3 E_{\text {beam }}=1.2 \mathrm{GeV}$ (for a 4 $\mathrm{GeV}$ beam energy), 75-90\% of the signal events over the full range of masses considered are kept and results in a factor of $\sim 30$ rejection of the background.

An additional kinematic handle for a missing momentum search comes from the electron transverse momentum $\left(p_{T}\right)$. Specifically, the electron in DM production will have a broad $p_{T}$ distribution. These are to be contrasted with the sharply falling $p_{T}$ distribution from bremsstrahlung, which (even after accounting for multiple scattering in a $10 \% X_{0}$ target) falls off as $1 / p_{T}^{3}$ for $p_{T} \gtrsim 4$ $\mathrm{MeV}$. As we will see below, bremsstrahlung-originated events dominate the background, so this kinematic difference is a powerful one.

\section{Background Processes}

Typically, an electron will propagate through the target without interacting, leaving a hard track in the tracker and a high-energy shower in the calorimeter. The case where the electron undergoes bremsstrahlung in the target resulting in a soft track below $E_{\text {cut }}=1.2 \mathrm{GeV}$ in the tracker occurs with a relative rate of $3 \times 10^{-2}$ per incident electron. These events have two showers in the ECal, with combined shower energy $\approx 4 \mathrm{GeV}$, separated by $1-2 \mathrm{~cm}$ or more depending on the electron energy. In rejecting these backgrounds, it becomes important to resolve the electron from the photon and measure the photon energy with high resolution. In this context, a photon that is very poorly measured can give rise to a fake dark photon signal.

A more challenging variation of hard bremsstrahlung is when, rather than producing a typical electromagnetic shower, the bremsstrahlung photon undergoes a rare process such as a photonuclear reaction or conversion to $\mu^{+} \mu^{-}$(or $\pi^{+} \pi^{-}$). Conversion to a pair of muons or pions not only occurs at a much smaller rate than photo-nuclear reactions, they are easier to reject given that they leave one or two "tracks" passing through the calorimeter.

The case where the photon undergoes a photo-nuclear reaction in the first few layers of the ECal occurs at a relative rate of $1.7 \times 10^{-5}$ per incident electron. The photo-nuclear processes initiated by $2.8-4 \mathrm{GeV}$ photons can result in a wide range of final states. When pions are produced in the interaction and escape the nucleus, they typically give rise to either "tracks" $\left(\pi^{ \pm}\right)$or substantial energy deposition $\left(\pi^{0}\right)$ in the ECal. In many cases, a large number of low-energy protons and neutrons are liberated from a heavy nucleus; some of the protons may deposit energy in the ECal, 
and some of the neutrons can be detected by the HCal. Two rare but important classes of these events are characterized by only two to three $\mathscr{O}(\mathrm{GeV})$, moderate-angle neutrons escaping from the nucleus, or a single forward $\sim 3 \mathrm{GeV}$ neutron. These events must be rejected by the HCal.

The same photo-nuclear reactions can also occur in the target. These events have $50 \times$ lower rate (relative to reactions in the ECal) because of the thin target material, and tracking provides additional handles to reject them, but they are also more difficult to detect calorimetrically because the effective angular coverage of the $\mathrm{HCal}$ is reduced. Electro-nuclear reactions in the target present a similar challenge given that they are kinematically similar to photo-nuclear reactions. However, they occur at a lower rate and have a broader $p_{T}$ distribution.

\section{Detector Concept}
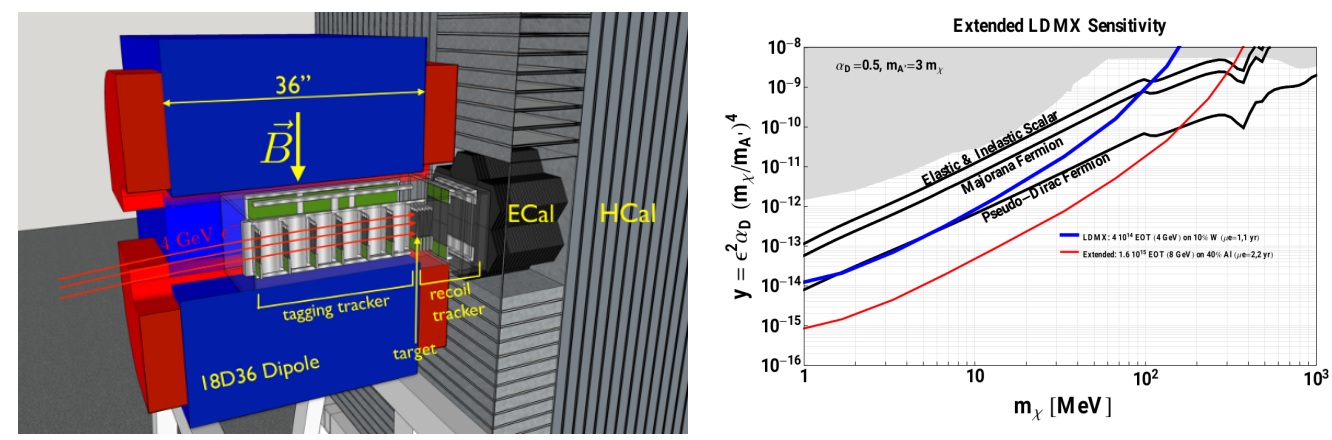

Figure 1: On the left, a cutaway overview of the LDMX detector showing, from left to right, the trackers and target inside the spectrometer dipole, the forward $\mathrm{ECal}$, and the $\mathrm{HCal}$. On the right, the blue line is the sensitivity of the "Phase LDMX discussed throughout these proceedings, conservatively assuming 0.5 background events. The red line is the sensitivity of an extended run using a sample of $1.6 \times 10^{15}, 8 \mathrm{GeV}$ electrons incident onto a $0.4 X_{0} \mathrm{Al}$ target and an average charge of $2 e^{-}$per bunch.

As described in Section 2, the signature of DM production used by LDMX involved (i) substantial energy loss by the electron, (ii) a potentially large transverse momentum kick, and (iii) the absence of any additional visible final-state particles that could carry away energy lost by the electron. Performing the measurement of each kinematic component on every incident electron requires a tracker and electromagnetic calorimetry (ECal) downstream of a $10 \%$ radiation-length $\left(0.1 X_{0}\right)$ target with a sensitive area that extends over the nominal beam axis. Placing the tracker in a weak magnetic field allows us to use some of the most striking of these events in which a soft, wide-angle recoil electron does not penetrate into the ECal. In addition, stray low-energy particles from beam halo must be rejected with very high efficiency, as these could mimic the DM signal. This motivates another tracker with stronger B-field upstream of the target that precisely measures the momentum and trajectory of the incoming beam electrons. While the energy and angle resolution requirements for LDMX are modest, the experiment poses two main challenges. First, every incident electron passes through the trackers and showers in the ECal; the detectors placed directly in the beam line must therefore contend with high radiation doses and a large event rate. In order to mitigate this issue, the beamspot for the experiment must be quite large - of order $10-100 \mathrm{~cm}^{2}$. Second, the requirement of an event veto for any additional visible final-state particles 
to take advantage of (iii), that is robust enough to handle a variety of rare backgrounds is not only an important physics performance driver for the tracking and ECal, but also calls for the addition of a dedicated hadron veto system surrounding the sides and back of the ECal.

These four detector systems - the "tagging tracker" upstream of the target, "recoil tracker" downstream, a radiation hard forward $\mathrm{ECal}$, and the hadronic veto system ( $\mathrm{HCal})$, form the majority of the LDMX experimental concept. To keep the detector compact and the field in the ECal minimal, we place the tagging tracker inside the bore of a dipole magnet and the recoil tracker in its fringe field. The layout for LDMX is illustrated in Figures 1.

\section{Conclusion}

The Light Dark Matter eXperiment (LDMX) described in these proceedings is designed to explore thermal relic dark matter over most of the viable sub-GeV mass range to a decisive level of sensitivity. The general strategy is to run the experiment in two phases. In "Phase I", LDMX will search for low-mass dark matter using a sample of $4 \times 10^{14}$ incident electrons, delivered onto a $10 \%$ radiation-length $\left(0.1 X_{0}\right)$ target with a $46 \mathrm{MHz}$ bunch spacing and average charge of one $e^{-}$per bunch. This would be followed by an "extended" or "Phase II" run with up to $\sim 10^{16}$ electrons on target and a beam energy of $8 \mathrm{GeV}$ of higher. The expected Phase I sensitivity of the LDMX experiment is shown in blue in Figure 1. The sensitivity of a representative Phase II scenario using a sample of $1.6 \times 10^{15}, 8 \mathrm{GeV}$ electrons incident on a $0.4 X_{0} \mathrm{Al}$ target and an average charge of $2 e^{-}$per bunch is also shown in red. Furthermore, using missing momentum measurements, LDMX will explore important new territory for secluded dark matter models, millicharge particles, invisibly decaying dark photons, axions and dark higgs particles. By extension, significant territory for SIMP, ELDER, asymmetric, and freeze-in dark matter scenarios will also be probed. Finally, using LDMX as a short baseline beam dump, provide sensitivity to displaced visibly decaying dark photons, axions, inelastic dark matter, dark higgs, and other long-lived dark sector particles. A detailed summary of the sensitivity of LDMX to all of these models can be found in [6]. As a multi-purpose experiment, LDMX, along with an appropriate set of complementary experiments, would therefore provide the foundation for a successful light dark matter program in the US or abroad.

\section{References}

[1] G. Bertone, D. Hooper and J. Silk, Phys. Rept. 405, 279 (2005) doi:10.1016/j.physrep.2004.08.031 [hep-ph/0404175].

[2] B. W. Lee and S. Weinberg, Phys. Rev. Lett. 39, 165 (1977). doi:10.1103/PhysRevLett.39.165

[3] J. Alexander et al., arXiv:1608.08632 [hep-ph].

[4] M. Battaglieri et al., arXiv:1707.04591 [hep-ph].

[5] T. ÃĚkesson et al. [LDMX Collaboration], arXiv:1808.05219 [hep-ex].

[6] A. Berlin, N. Blinov, G. Krnjaic, P. Schuster and N. Toro, arXiv:1807.01730 [hep-ph]. 